\title{
A first step towards a global nomogram to predict disease progression for men on active surveillance
}

\author{
Mieke Van Hemelrijck ${ }^{1}$, Xinge $\mathrm{Ji}^{2}$, Jozien Helleman ${ }^{3}$, Monique J. Roobol ${ }^{3}$, Daan Nieboer ${ }^{3}$, \\ Chris Bangma $^{3}$, Mark Frydenberg ${ }^{4}$, Antti Rannikko ${ }^{5}$, Lui Shiong Lee ${ }^{6}$, Vincent Gnanapragasam ${ }^{7}$, \\ Michael W. Kattan'; Movember Foundation's Global Action Plan Prostate Cancer Active Surveillance \\ (GAP3) Consortium*
}

${ }^{1}$ Translational Oncology \& Urology Research (TOUR), School of Cancer and Pharmaceutical Sciences, King's College London, London, UK; ${ }^{2}$ Department of Quantitative Health Sciences, Cleveland Clinic, Cleveland, OH, USA; ${ }^{3}$ Department of Urology, Erasmus University Medical Center, Rotterdam, The Netherlands; ${ }^{4}$ Cabrini Institute, Malvern, Australia; ${ }^{5}$ Department of Urology, Helsinki University and Helsinki University Hospital, Helsinki, Finland; ${ }^{6}$ Department of Urology, Sengkang General Hospital and Singapore General Hospital, Singapore, Singapore; ${ }^{7}$ Academic Urology Group, Department of Surgery and Oncology, University of Cambridge, Cambridge, UK

Contributions: (I) Conception and design: MW Kattan, MJ Roobol, J Helleman, D Nieboer, M Van Hemelrijck, X Ji; (II) Administrative support: J Helleman, MJ Roobol; (III) Provision of study materials or patients: J Helleman, MJ Roobol, D Nieboer, C Bangma, M Frydenberg, A Rannikko, LS Lee, V Gnanapragasam, MW Kattan, Movember Foundation's Global Action Plan Prostate Cancer Active Surveillance (GAP3) Consortium; (IV) Collection and assembly of data: D Nieboer; (V) Data analysis and interpretation: D Nieboer, X Ji, M Van Hemelrijck, MJ Roobol, MW Kattan; (VI) Manuscript writing: All authors; (VII) Final approval of manuscript: All authors.

Correspondence to: Prof. Mieke Van Hemelrijck. Translational Oncology \& Urology Research (TOUR), School of Cancer and Pharmaceutical Sciences, King's College London, Guy's Hospital, 3rd Floor Bermondsey Wing, London SE1 9RT, UK. Email: Mieke.vanhemelrijck@kcl.ac.uk.

Background: Signs of disease progression (28\%) and conversion to active treatment without evidence of disease progression (13\%) are the main reasons for discontinuation of active surveillance (AS) in men with localised prostate cancer (PCa). We aimed to develop a nomogram to predict disease progression in these patients.

Methods: As a first step in the development of a nomogram, using data from Movembers' GAP3 Consortium ( $n=14,380)$, we assessed heterogeneity between centres in terms of risk of disease progression. We started with assessment of baseline hazards for disease progression based on grouping of centres according to follow-up protocols [high: yearly; intermediate: 2 yearly; and low: at year 1, 4 \& 7 (i.e., PRIAS)]. We conducted cause-specific random effect Cox proportional hazards regression to estimate risk of disease progression by centre in each group.

Results: Disease progression rates varied substantially between centres [median hazard ratio (MHR): 2.5]. After adjustment for various clinical factors (age, year of diagnosis, Gleason grade group, number of positive cores and PSA), substantial heterogeneity in disease progression remained between centres.

Conclusions: When combining worldwide data on AS, we noted unexplained differences of disease progression rate even after adjustment for various clinical factors. This suggests that when developing a global nomogram, local adjustments for differences in risk of disease progression and competing outcomes such as conversion to active treatment need to be considered.

Keywords: Prostate cancer (PCa); active surveillance (AS); discontinuation; worldwide; nomogram

Submitted Jul 09, 2020. Accepted for publication Nov 12, 2020.

doi: $10.21037 /$ tau-20-1082

View this article at: http://dx.doi.org/10.21037/tau-20-1082

* Available online: https://cdn.amegroups.cn/static/public/TAU-20-1082-GAP3.pdf 


\section{Introduction}

Using data from the Movember GAP3 Consortium, the largest centralised prostate cancer $(\mathrm{PCa})$ active surveillance (AS) database to date, we have previously shown that after 5 years of follow-up, about $56.4 \%$ of men who started on AS were still on AS (1). We noted substantial variation by centre, but signs of disease progression ( $27.5 \%$ of men) and conversion to active treatment without evidence of disease progression (12.8\% of men) were the main reasons for discontinuation.

A wide variety of predictive factors, including clinical and pathological factors, multi-parametric MRI (mpMRI) data, variability in biopsy timing, and nomograms are currently proposed for men with low-risk PCa on AS $(2,3)$. For example, Wang et al. compared the Kattan, Steyerberg, Nakanishi and Chun nomograms to calculate the likelihood of indolent disease as well as various forms of progression in 273 patients meeting low-risk criteria who were managed by AS and who underwent multiple biopsies and/or delayed radical prostatectomy (4). Based on this fairly small sample size, it was concluded that existing nomograms have only modest accuracy in predicting the outcomes of patients undergoing AS.

Moreover, a recent national survey in the US evaluated the current use of prediction tools and nomograms for localised PCa based on clinical practice from radiation oncologists and urologist. A fifth of specialists reported not using any such tools in clinical practice, mainly due to the low use of AS for low-risk PCa (5). It is exactly for these reasons that the Movember Foundation launched its Global Action Plan Prostate Cancer Active Surveillance initiative (GAP3) in 2014. Its primary goal is to create a global consensus with uniform guidelines on the selection and monitoring of men with low-risk PCa (6).

Following the assessment of reasons for discontinuation, we now aimed to develop a nomogram to be used as a prediction tool for outcomes in men with localised PCa on AS. We present the following article in accordance with the MDAR reporting checklist (available at http://dx.doi. org/10.21037/tau-20-1082).

\section{Methods}

\section{Study population}

By combining patient data from established AS cohorts worldwide, the global GAP3 database was created between 2014 and 2016. In addition to ethical approval for sharing digital patient data in a centralized global database, inclusion criteria required an active registry of AS patients over the last 2 years or more, including at least $\sim 50$ patients annually. To date, 25 centres from the USA, Canada, Australasia, the UK and Europe fulfilled the requirements for participation and joined the initiative (6) resulting in data for a total of 15,101 men on AS (GAP3 database v2.3.2). Each institution obtained institution ethical approval and signed a Movember End User License Agreement, Access Rights Principles Agreement, and the commonly agreed upon GAP3 analytical plan for the use of routinely collected anonymised clinical data. The study has conformed to the provisions of the Declaration of Helsinki (as revised in 2013). Details of ethical approval for each individual institution can be found in our cohort profile (6).

The AS inclusion criteria for the participating centres have been shown previously (1); despite the variation in protocols, most centres agreed that the most suitable patients for AS are those with age $>18$, pre-treatment clinical stage $\mathrm{T} 1-\mathrm{T} 2$, serum PSA $\leq 10 \mathrm{ng} / \mathrm{mL}$, a biopsy Gleason grade group of 1 or 2, and a maximum of two tumour-positive biopsy core samples. An overview of contemporary worldwide AS practices across the world (and included in GAP3) can also be found in the systematic review by Kinsella et al. (7) and the cohort profile of the GAP3 database (8).

Apart from the inclusion criteria, there is also some variation in follow-up protocols. However, almost all protocols recommended serial measurements (with a variation in time-intervals) of serum PSA levels, digital rectal examination (DRE) and surveillance biopsy sampling in order to identify pathological progression. An overview of the AS follow-up protocols in GAP3 can be found in our previous publications $(1,6)$. For the current analyses, we have grouped centres according to similarities in follow-up protocols (high, intermediate, and low) - the term "groups" in the results section hence refers to these different AS follow-up protocols. The high group includes those centres that performed a biopsy on an annual basis (Johns Hopkins, Atlanta, Lille, Singapore, Kagawa, UCSF, and MD Anderson), whereas the intermediate group includes those centres where the follow-up biopsy was taken about 2 years after AS initiation (Memorial Sloan Kettering, Calgary, Baden, Cambridge, Valencia, MUSIC, Dublin, and Milan-PRIAS), and the low group refers to those centres that followed the PRIAS criteria, i.e., 1, 4, and 7 years after AS initiation (Erasmus, Vancouver, Helsinki, Malmo, Melbourne, and Toronto, and Milan-SAINT). Hence, a 
total of 14,380 men were included in the analyses. The median age of the total population was 65 with IQR $60-70$.

With respect to indicators of disease progression, we have previously (1) described that there is heterogeneity in recording across centres. We therefore used the following coding for defining signs of disease progression: 'convert to watchful waiting', 'clinical progression', 'pathological progression', 'clinical and pathological progression', 'PSA progression (PSA-DT <3 years)', 'other PSA kinetics', 'Patient choice/anxiety', 'doctors anxiety', 'radiological progression', 'died', 'lost to FU', 'other/unknown' or 'still on active surveillance'. If the reason for discontinuation of AS was classified as 'other/unknown' but the 'pathological progression status' was 'Gleason grade group 3 or higher' or the 'clinical progression status' was 'cT3 or higher' or 'PSA progression status' was 'PSA $>20$ ', the reason for discontinuation of AS was also categorised as signs of disease progression. Thus, similar to our previous publication (1), "disease progression" can refer to risk reclassification or disease progression as such and was used as the main outcome of interest in the current project. Conversion to active treatment, watchful waiting, or death were considered as competing risks.

\section{Statistical methods}

The main aim of the study was to create a nomogram to predict disease progression when on AS for PCa. First, we conducted cause-specific Cox proportional hazards regression to estimate the hazard ratio (HR) of disease progression and competing risks (watchful waiting, conversion to active treatment, or death) based on centre (i.e., exposure variable). The heterogeneity of prevalence of clinical factors and their relationship to disease progression was studied by dichotomising the clinical factors and modelling the univariable HRs of clinical factors by centre. Following the univariable analysis by centre, we then adjusted for age, year of diagnosis, Gleason grade group, number of positive cores, and PSA. Johns Hopkins was selected as the reference centre because it is one of the largest centres in the study and its patients characteristics are close to the average.

To further investigate heterogeneity in baseline upgrading risk between centres we estimated Cox frailty models. These frailty models include a random effect term for centre and the difference in upgrading risk between centres is parameterized using the variance of the frailty term. To aid the interpretation of the results we transformed the variance term to the median hazard ratio (MHR) (9). The MHR can be interpreted as the MHR of disease progression when moving a patient from a randomly chosen centre to a randomly chosen centre with a higher disease progression risk (Or comparing a patient with the same characteristics between two randomly chosen centres). We calculated the MHR using a frailty model not including any patient and tumour characteristics and investigated whether differences in included patients could partially explain the observed heterogeneity by adjusting for characteristics included in the previous analyses.

All statistical analyses were performed in R. Statistical codes are available upon request.

\section{Results}

Tables 1-3 show reasons for AS discontinuation the three groups of centres based on their study protocol for follow-up. In the centres that performed a biopsy on an annual basis (Table 1) and the centres that followed the PRIAS criteria (Table 3), the majority of patients in most of the centres discontinued due to treatment for disease progression. In the centres where the follow-up biopsy was taken more than a year after AS initiation (Table 2), the main reason for discontinuation was either treatment for disease progression or conversion to watchful waiting or active treatment (without evidence of progression).

We performed univariable heterogeneity analysis for disease progression whilst considering all other reasons for discontinuation as censoring events. Figure 1 illustrates this graphically by showing the distribution of HRs for both conversion to active treatment as well as progression. In each group, there was considerable variability in the disease progression rate (MHR: 2.5, Figure 2).

Next, we assessed how clinical factors such as age, year of diagnosis, Gleason grade group $(<1 \geq 2)$, number of positive cores $(</ \geq 2)$, and PSA could explain the different disease progression rates in each centre. After adjustment for these clinical factors, the heterogeneity in risk of disease progression remained between the centres (Figure 2).

\section{Discussion}

When combining worldwide cohorts of men on AS, we noted unexplained differences in disease progression rates between centres (MHR: 2.5). After adjustment for various clinical factors (age, year of diagnosis, Gleason grade group, number of positive cores and PSA), substantial 
Table 1 Overview of reasons for AS discontinuation in those centres that performed a biopsy on an annual basis (high group)

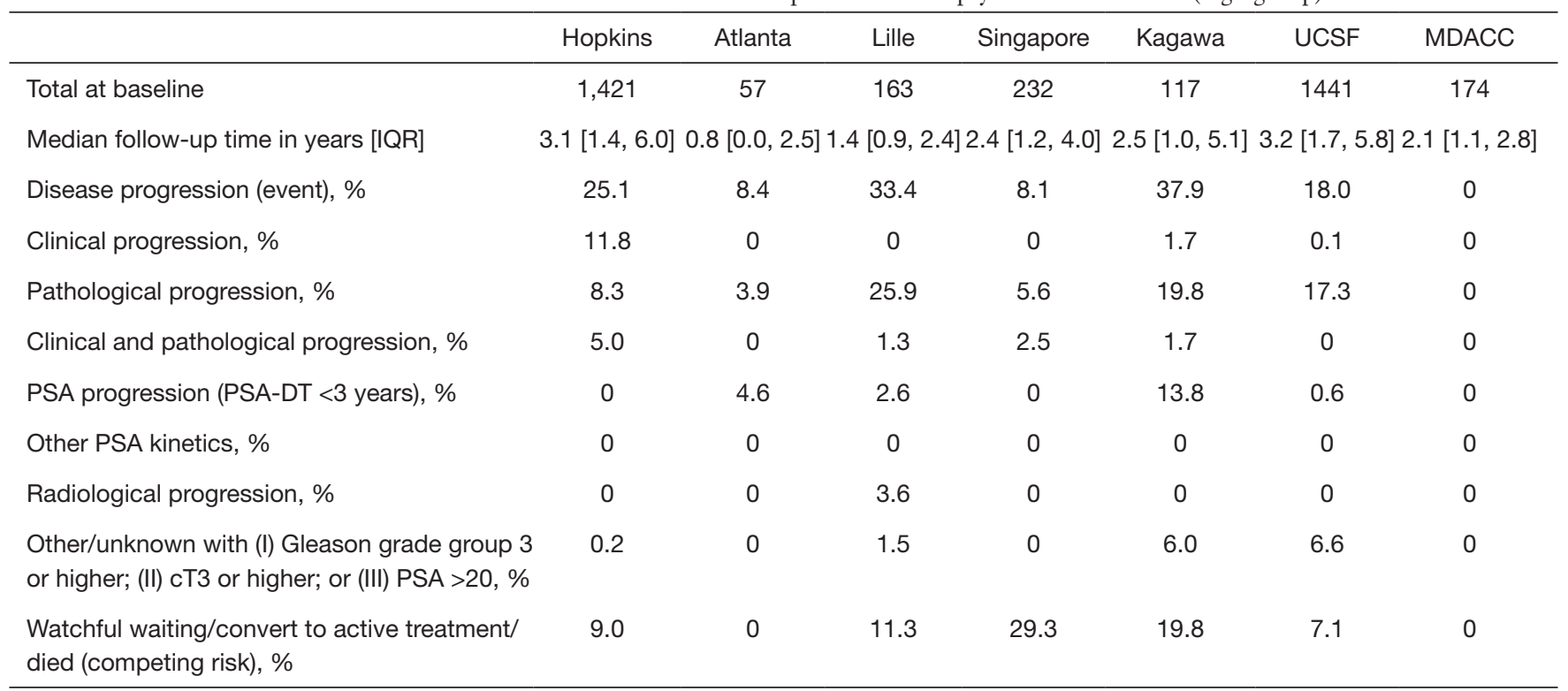

Data shown as 3-year cumulative incidence. AS, active surveillance.

Table 2 Overview of reasons for AS discontinuation in those centres where the follow-up biopsy was taken more than a year after AS initiation (intermediate group)

\begin{tabular}{|c|c|c|c|c|c|c|c|c|}
\hline & MSKCC & Calgary & Baden & Cambridge & Valencia & MUSIC & Dublin & Milan-SAINT \\
\hline Median follow-up time in years [IQR] & $\begin{array}{c}4.4 \\
{[1.9,6.3]}\end{array}$ & $\begin{array}{c}2.3 \\
{[1.2,3.9]}\end{array}$ & $\begin{array}{c}4.0 \\
{[2.2,6.4]}\end{array}$ & $\begin{array}{c}2.1 \\
{[1.1,3.5]}\end{array}$ & $\begin{array}{c}2.0 \\
{[0.8,3.7]}\end{array}$ & $\begin{array}{c}0.8 \\
{[0.3,1.5]}\end{array}$ & $\begin{array}{c}1.4 \\
{[1.0,2.0]}\end{array}$ & $\begin{array}{c}2.2 \\
{[1.1,4.0]}\end{array}$ \\
\hline Disease progression (event), \% & 3.9 & 16.3 & 17.6 & 6.5 & 24.7 & 6.9 & 0 & 41.8 \\
\hline Clinical and pathological progression, \% & 0.1 & 0 & 0 & 0.9 & 0 & 0 & 0 & 13.9 \\
\hline PSA progression (PSA-DT <3 years), \% & 0.2 & 0 & 0 & 0 & 0.4 & 0.5 & 0 & 3.4 \\
\hline Other PSA kinetics, \% & 0 & 0 & 0 & 0 & 0 & 0 & 0 & 0 \\
\hline $\begin{array}{l}\text { Watchful waiting/convert to active } \\
\text { treatment/died (competing risk), } \%\end{array}$ & 17.4 & 7.0 & 10.8 & 4.8 & 12.0 & 28.5 & 39.5 & 14.3 \\
\hline
\end{tabular}

Data shown as 3-year cumulative incidence. AS, active surveillance.

heterogeneity in disease progression remained between centres.

The current European guidelines suggest that a large proportion of men with localized PCa do not require immediate radical treatment, but can be safely monitored using AS (10). Yet, despite the potential to monitor this low-risk PCa long-term with very little risk of disease progression ( $<0.03 \%$ over 15 years) (7), uptake of AS varies 
Table 3 Overview of reasons for AS discontinuation in those centres that followed the PRIAS criteria (low group)

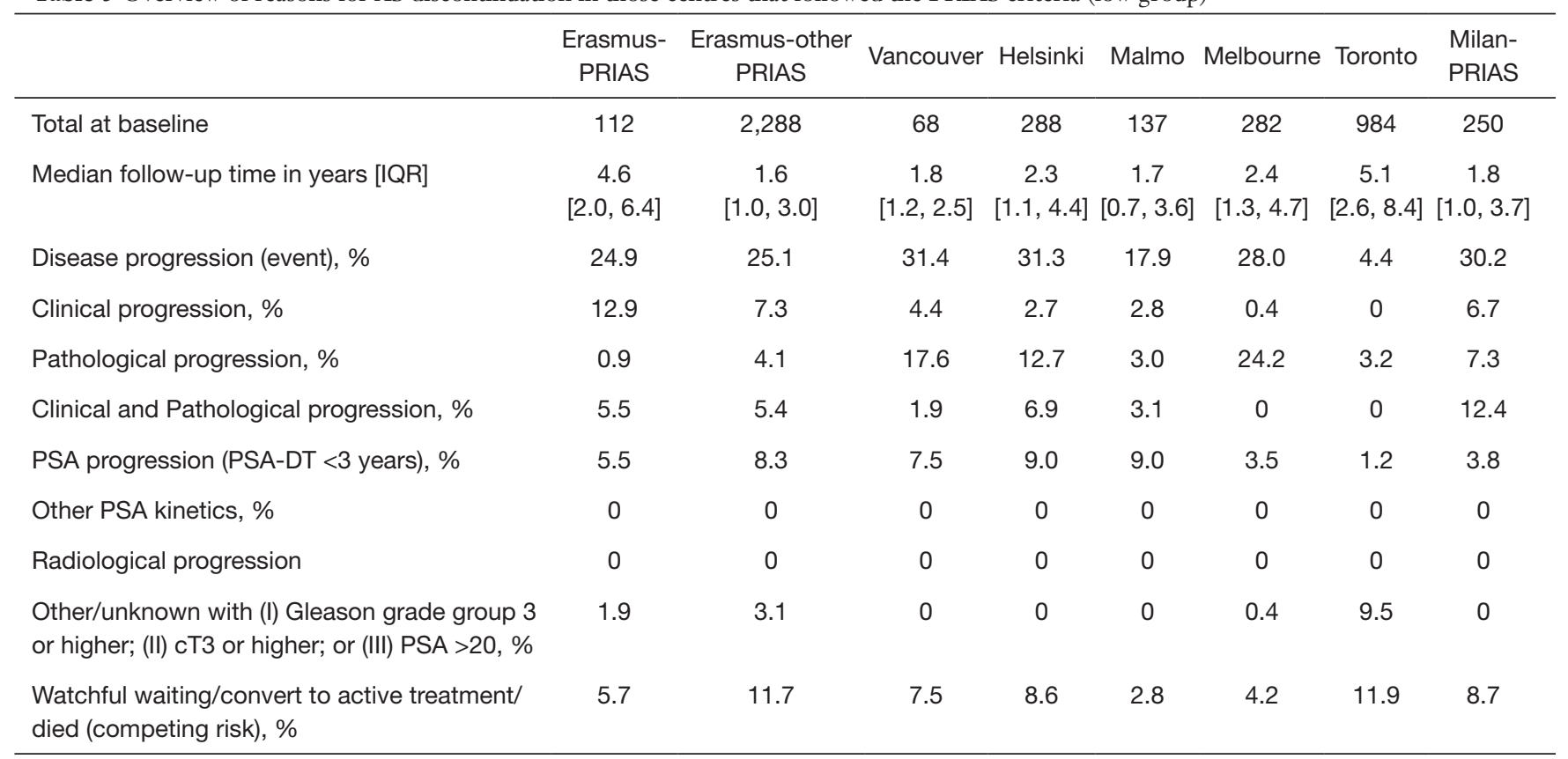

Data shown as 3-year cumulative incidence. AS, active surveillance.

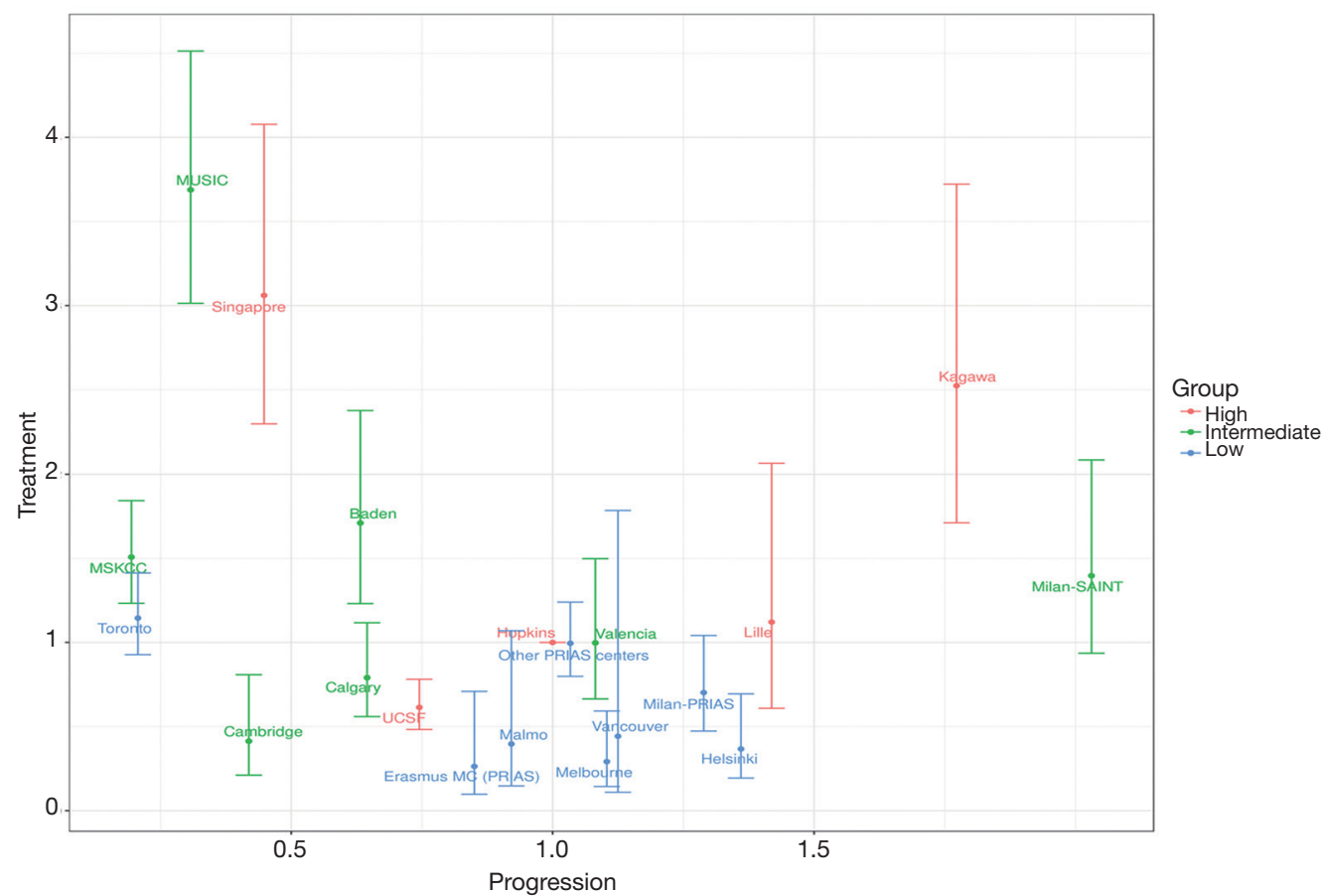

Figure 1 Univariable heterogeneity analysis, as quantified by hazard ratios (HRs), for disease progression (X-axis) and conversion to active treatment (Y-axis), whilst considering all other reasons for discontinuation as competing risks. 


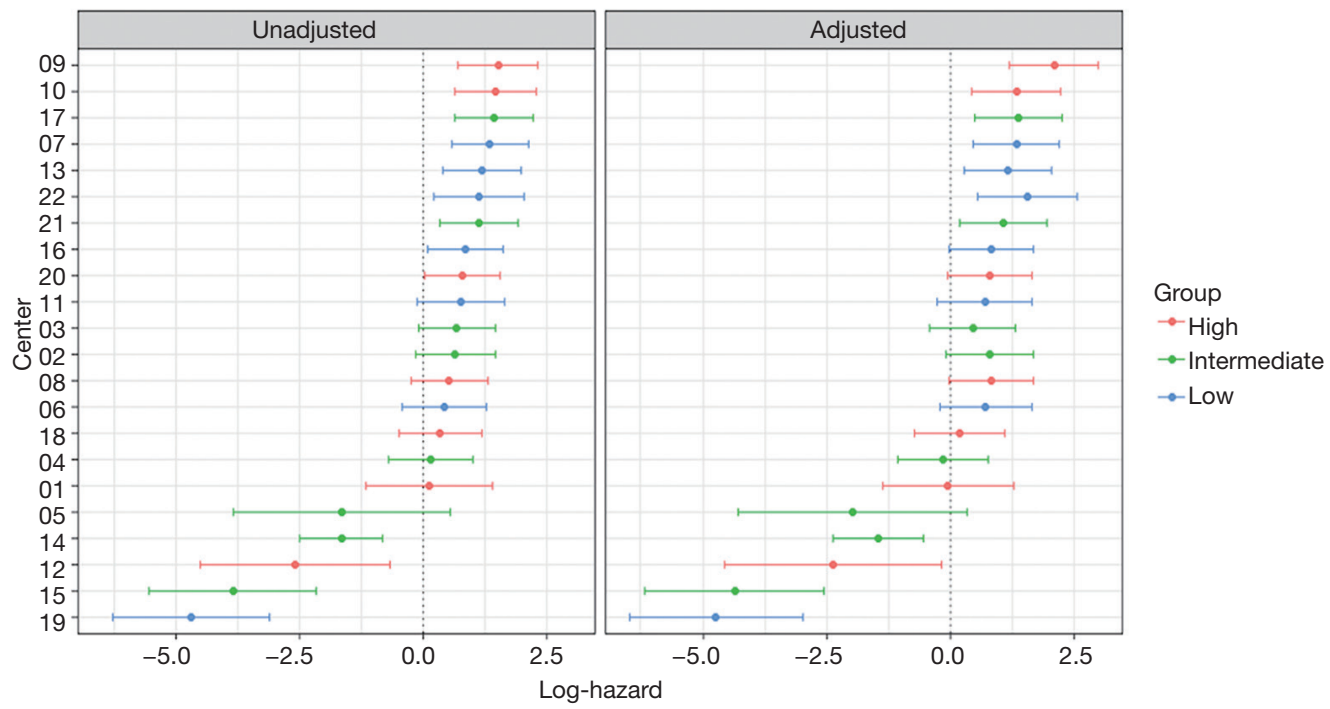

Figure 2 Log-hazard and 95\% confidence intervals (X-axis) for disease progression based on centre. The adjusted model takes into account age, year of diagnosis, Gleason grade group, and number of positive cores.

across countries and practices (11) and drop-out is reported to be as high as $38 \%$ - even where there is no evidence of disease progression (7). As mentioned previously, in our own GAP3 cohort we found that after 5 years of followup, about $43 \%$ of men who started on AS discontinued (1). Conversion to active treatment without evidence of disease progression was indeed one of the main reasons for discontinuation (12.8\%). Low acceptability of AS may result into overtreatment, unnecessary adverse events, and a higher health economic burden-hence, justifying the need for the development of a worldwide nomogram.

There is a need to develop a nomogram to predict disease progression in men suitable for AS with an optimal balance between sensitivity and specificity as current nomograms have only been found to show limited accuracy in predicting outcomes of these men (4). The various risk prediction tools available to date have shown potential, though all in limited settings. For example, Iremashvili et al. compared the probabilities of pathologically relatively aggressive disease using the Partin and Dinh risk tables and Kattan, Truong, and Kulkarni nomograms using a cohort of $326 \mathrm{PCa}$ patients. It was found that the predictions of Partin and Dinh tables were not associated with biopsy progression, whereas the predictive value of Kattan and Truong nomograms was higher when compared with the other tools-though only for the first and second surveillance biopsies. The latter two nomograms were also able to identify low and high-risk subgroups, though the
Kattan nomogram was found to show better correlation with the observed progression rate (12). Hence, using a large worldwide database including various centres, we aimed to develop a more widely applicable nomogram.

As shown in Figure 1, there was a large discrepancy between centres in terms of baseline hazards of disease progression. This is problematic when trying to develop a global nomogram and a better understanding of these observed differences is required before these can be taken into account in the mathematical models underlying a nomogram. An in-depth assessment of inclusion criteria and competing risks (e.g., watchful waiting, conversion to active treatment, or death) of the various centres is needed to help disentangle these baseline risks of disease progression. However, it is interesting to note that the relative effects of patient characteristics on risk of disease progression were fairly stable between centres (as shown in Figure 2). Upon adjustment for important known clinical indicators of disease progression, the heterogeneity between centres remained. These findings combined therefore indicate that it is possible to develop a global nomogram in future if local adjustments for differences in risk of disease progression and competing risks can be accounted for. Differences in disease progression rates can be incorporated by first collecting patient information and progression rates in a new centre, and subsequently using the collected data to update a global nomogram by re-estimating the baseline hazard to adjust the average predicted reclassification rate. 
As already shown in our previous publications (1,6,13-16), Movember's GAP3 database provides a unique opportunity to assess worldwide data in the setting of AS for men with localised PCa. However, some limitations exist because not all centres could be included in our analyses due to the lack of information on data for discontinuation. Moreover, there is heterogeneity in study protocols and data collection across centres. Nevertheless, our findings show that this heterogeneity in clinical factors and followup protocols does not explain the heterogeneity in risk of disease progression as such (Figure 2). It is therefore of interest to further disentangle this real-world data resource as it can help the development of a global nomogram. The next update of the GAP3 database will help address this next step of the development of a global nomogram as the database will then also contain information on imaging (MRI), molecular (genomics) markers, and patient-related outcomes.

\section{Conclusions}

Our findings of unexplained differences in baseline hazards for disease progression and consistent effects of clinical predictors between centres in the GAP3 database indicate that it is possible to develop a global nomogram in future. A next step involves the development of mathematical methods for local adjustments for differences in risk of disease progression and competing risks (e.g., watchful waiting, conversion to active treatment, or death) based on further examination of the underlying reasons for this heterogeneity.

\section{Acknowledgments}

Funding: This work was supported by the Movember Foundation.

\section{Footnote}

Reporting Checklist: The authors have completed the MDAR reporting checklist. Available at http://dx.doi.org/10.21037/ tau-20-1082

Data Sharing Statement: Available at http://dx.doi. org/10.21037/tau-20-1082

Peer Review File: Available at http://dx.doi.org/10.21037/ tau-20-1082
Conflicts of Interest: All authors have completed the ICMJE uniform disclosure form (available at http://dx.doi. org/10.21037/tau-20-1082). The authors have no conflicts of interest to declare.

Ethical Statement: The authors are accountable for all aspects of the work in ensuring that questions related to the accuracy or integrity of any part of the work are appropriately investigated and resolved. The study has conformed to the provisions of the Declaration of Helsinki (as revised in 2013).

Open Access Statement: This is an Open Access article distributed in accordance with the Creative Commons Attribution-NonCommercial-NoDerivs 4.0 International License (CC BY-NC-ND 4.0), which permits the noncommercial replication and distribution of the article with the strict proviso that no changes or edits are made and the original work is properly cited (including links to both the formal publication through the relevant DOI and the license). See: https://creativecommons.org/licenses/by-nc-nd/4.0/.

\section{References}

1. Van Hemelrijck M, Ji X, Helleman J, et al. Reasons for discontinuing active surveillance: assessment of 21 centres in 12 countries in the Movember GAP3 Consortium. Eur Urol 2019;75:523-31.

2. Lai WS, Gordetsky JB, Thomas JV, et al. Factors predicting prostate cancer upgrading on magnetic resonance imaging-targeted biopsy in an active surveillance population. Cancer 2017;123:1941-8.

3. Wang JH, Downs TM, Jason Abel E, et al. Prostate biopsy in active surveillance protocols: immediate re-biopsy and timing of subsequent biopsies. Curr Urol Rep 2017;18:48.

4. Wang SY, Cowan JE, Cary KC, et al. Limited ability of existing nomograms to predict outcomes in men undergoing active surveillance for prostate cancer. BJU Int 2014;114:E18-24.

5. Gershman B, Maroni P, Tilburt JC, et al. A national survey of radiation oncologists and urologists on prediction tools and nomograms for localized prostate cancer. World J Urol 2019;37:2099-108.

6. Bruinsma SM, Zhang L, Roobol MJ, et al. The Movember Foundation's GAP3 cohort: a profile of the largest global prostate cancer active surveillance database to date. BJU Int 2018;121:737-44.

7. Kinsella N, Helleman J, Bruinsma S, et al. Active 
surveillance for prostate cancer: a systematic review of contemporary worldwide practices. Transl Androl Urol 2018;7:83-97.

8. Bruinsma SM, Bangma CH, Carroll PR, et al. Active surveillance for prostate cancer: a narrative review of clinical guidelines. Nat Rev Urol 2016;13:151-67.

9. Austin PC, Wagner P, Merlo J. The median hazard ratio: a useful measure of variance and general contextual effects in multilevel survival analysis. Stat Med 2017;36:928-38.

10. Cooperberg MR, Carroll PR. Trends in management for patients with localized prostate cancer, 1990-2013. JAMA 2015;314:80-2.

11. Auffenberg GB, Lane BR, Linsell S, et al. Practice- vs physician-level variation in use of active surveillance for men with low-risk prostate cancer: implications for collaborative quality improvement. JAMA Surg 2017;152:978-80.

12. Iremashvili V, Manoharan M, Kava BR, et al. Predictive models and risk of biopsy progression in active surveillance patients. Urol Oncol 2017;35:37.e1-8.

Cite this article as: Van Hemelrijck M, Ji X, Helleman J, Roobol MJ, Nieboer D, Bangma C, Frydenberg M, Rannikko A, Lee LS, Gnanapragasam V, Kattan MW; Movember Foundation's Global Action Plan Prostate Cancer Active Surveillance (GAP3) Consortium. A first step towards a global nomogram to predict disease progression for men on active surveillance. Transl Androl Urol 2021;10(3):1102-1109. doi: 10.21037/tau-20-1082
13. Kalapara AA, Verbeek JFM, Nieboer D, et al. Adherence to active surveillance protocols for low-risk prostate cancer: results of the Movember Foundation's Global Action Plan Prostate Cancer Active Surveillance Initiative. Eur Urol Oncol 2020;3:80-91.

14. Drost FH, Nieboer D, Morgan TM, et al. Predicting biopsy outcomes during active surveillance for prostate cancer: external validation of the canary prostate active surveillance study risk calculators in five large active surveillance cohorts. Eur Urol 2019;76:693-702.

15. van der Kwast TH, Helleman J, Nieboer D, et al. Consistent biopsy quality and Gleason grading within the global active surveillance global action plan 3 initiative: a prerequisite for future studies. Eur Urol Oncol 2019;2:333-6.

16. Bruinsma SM, Roobol MJ, Carroll PR, et al. Expert consensus document: Semantics in active surveillance for men with localized prostate cancer - results of a modified Delphi consensus procedure. Nat Rev Urol 2017;14:312-22. 